\title{
Difficulties in the Diagnosis of Gaucher Disease in a Low-Income Country: A Case Report from Mozambique
}

Journal of Inborn Errors of Metabolism \& Screening 2021, Volume 9: e20200022 DOI: https://doi.org/10.1590/2326-4594JIEMS-2020-0022

\author{
Félix Pinto' ${ }^{1}$, Ema Nassone ${ }^{2}$, Muhammad Ismail'², \\ Astrilde Jamisse ${ }^{3}$, Francyne Kubaski ${ }^{4,5}$, Ana Carolina Brusius-Facchin ${ }^{4}$, \\ Roberto Giugliani, ${ }^{4,5,6}$, Luís Madeira ${ }^{1}$ and Fabíola Fernandes ${ }^{7}$
}

\begin{abstract} the substrate glucosylceramide. pathogenic variant p.Arg87Trp (R48W) in homozygosis.

Discussion and Conclusion: To the best of our knowledge, this report describes the first case of GD type 1 confirmed via biochemical and molecular genetic testing in Mozambique. As awareness of the GD and rare genetic diseases among Mozambican health professionals is very limited, and resources for diagnosis are scarce in the national health system, it is possible that other cases remain undiagnosed in this low-income country.
\end{abstract}

Introduction: Gaucher disease (GD) is one of the common lysosomal storage disorder (LSD) with an estimated frequency of one in 40,000 newborns globally. GD is an autosomal recessive condition, which results from mutations in the GBA1 gene, causing partial or complete deficiency of $\beta$-glucocerebrosidase enzyme activity, which leads to the widespread accumulation of

Aims: This report presents different challenges of clinical management and communication between medical specialties to reach diagnose of any rare disease in Mozambique, a low-income country, which health system has limited infrastructure, trained personnel, and budget for diagnosis and to provide treatment for rare genetic disorders such as GD.

Case Presentation: The patient was a 15-year old black female patient of Mozambican nationality born from non-consanguineous parents. Three of the four patient's siblings were healthy; one sister had died of a disease with a similar clinical features. Our patient presented with abdominal distention and hepatosplenomegaly. Blood tests revealed pancytopenia and a high level of ferritin. Liver biopsy and histologic examination revealed infiltration of the splenic parenchyma and portal area of the liver as well as enlarged histiocytic cells with granular cytoplasm. Magnetic resonance imaging showed liver enlargement, changes in the femoral heads without osteonecrosis, a pathological fracture of the third thoracic vertebrae (T3), with absence of brain and spinal cord neurological abnormalities. The biochemical investigation disclosed low levels of $\beta$-glucocerebrosidase $(0.223 \mathrm{nmol} / \mathrm{h} / \mathrm{ml}$; normal: above 0.98$)$ and increased levels of lyso-Gb1 $(0.43 \mu \mathrm{g} / \mathrm{ml}$; normal: up to 0.003$)$. Genotyping of the GBA1 gene indicated the presence of the

Keywords: Gaucher Disease, Lysosomal disease, $\beta$-glucocerebrosidase, lysoGb1, GBA1, Mozambique.

\section{Introduction}

Gaucher Disease (GD) is one of the most common lysosomal disorders[1,2], with incidence figures that vary according to region and to population groups[2-4], and an estimated frequency of one in 40,000 individuals[1,2]. GD

\footnotetext{
${ }^{1}$ Hospital Central de Maputo, Serviço de Genética Médica, Maputo, Mozambique. ${ }^{2}$ Hospital Central de Maputo, Serviço de Gastroenterologia, Maputo, Mozambique.

${ }^{3}$ Hospital Central de Maputo, Serviço de Radiologia e Imagiologia, Maputo, Mozambique.

${ }^{4}$ Hospital de Clínicas de Porto Alegre, Serviço de Genética Médica, Rede DLD Brasil, Porto Alegre, RS, Brazil.

${ }^{5}$ Universidade Federal do Rio Grande do Sul, Programa de Pós-Graduação em Genética e Biologia Molecular, Porto Alegre, RS, Brazil.

${ }^{6}$ Universidade Federal do Rio Grande do Sul, Departamento de Genética, Porto Alegre, RS, Brazil.

${ }^{7}$ Hospital Central de Maputo, Serviço de Anatomia Patológica, Maputo, Mozambique.
}

Received November 20, 2020. Revised form January 19, 2021. Accepted for publication January 22, 2021.

Corresponding Author:

Félix Pinto, Hospital Central de Maputo, Serviço de Genética Médica, Maputo, Mozambique.

Email: fpdpinto@gmail.com 
is an autosomal recessive condition that results from mutations in the GBA1 gene (located in chromosome 1q21), causing low levels of $\beta$-glucocerebrosidase enzyme activity $[3,5]$. This results in widespread accumulation of the substrate glucosylceramide [5-7], which in normal circumstances is converted by the enzyme into glucose and ceramide[6].

The clinical features are diverse according to the type of $\mathrm{GD}[6,8,9]$. In type 1 , the accumulation of substrate results in the enlargement of the liver and spleen, fatigue, growth retardation, delayed puberty, bleeding disorders, pancytopenia, and painful bone crisis related to vascular diseases or mechanic effects[6,8,10]. Complicated cases can evolve with hepatocellular carcinoma[11], sensorial system disease[12], central nervous system damage and ultimately Parkinson disease[13,14], myelodysplasia due to bone infiltration or leukaemia[15]. Type 2 is a catastrophic disease, with acute somatic and neurological involvement and early death, and type 3 is an intermediate between 1 and 2, with somatic and neurological manifestations that can impact patient development as well as in the quality of life and life expectancy[3,5].

GD diagnosis is in average made at 10 years of age, given difficulties recognising symptoms[6,8]. Confirmation of GD is made by the measurement of beta glucocerebrosidase enzyme activity in blood. Biomarkers such as chitotriosidase, CCL18 and LysoGb1 are useful in the diagnostic investigation, and identification of mutations in the GBA1 gene can provide further diagnostic confirmation and help to predict the phenotype $[4,8,16]$.

Mozambique is a low-income country that ranks in the bottom 10 positions of the global human development index $\left(180^{\text {th }}\right)[17]$, where approximately $60 \%$ of the population has access to basic healthcare services. Malnutrition, tuberculosis, HIV, malaria, among other prevalent problems strain a fragile healthcare system and consume more than $60 \%$ of the state healthcare budget, affecting access and quality of primary healthcare services[18]. With the exception of the main national referral hospital, Hospital Central de Maputo (HCM), there is no laboratory infrastructure to diagnosis genetic disorders such as
GD, and treatment options such as enzyme replacement therapy or substrate inhibition therapy are extremely limited[3,6].

Here we report on a patient who was diagnosed with GD at HCM.

\section{Case Presentation}

The patient is a 15 -year-old black female. She was born in Mozambique from non-consanguineous parents. Three of the four patient's siblings were healthy and one sister had died at 5 years of age after splenectomy surgery, with clinical features of GD (Figure 1). There was no previous family history of GD or similar conditions reported.

Her symptoms began when she was 5 years old when their parents noticed progressive abdominal distension and sought medical care. The Pediatric Hematology and Oncology service observed the patient first time at 11 years of age and had a bone marrow aspirate, which showed alterations suggestive of idiopathic thrombocytopenic purpura, without other abnormalities noticed.

Afterwards the patient was sent to the gastroenterology service, where endoscopy was performed. She was noted to have erosive gastritis and was treated with proton pump inhibitors with resolution of findings on endoscopic follow up. She was referred back to the paediatric department for continued followup. At this point, she did not have neurologic or psychomotor alterations.

Two years later, at the age of 13 , she was admitted to the pediatric emergency service with a recent history of two episodes of epistaxis and abdominal distension. She was diagnosed with hypersplenism and underwent splenectomy (the removed, enlarged spleen weighed $2.7 \mathrm{~kg}$ ). Histologic examination of the spleen revealed an enlarged vacuoles suggestive of GD (this finding only came to the authors' attention after diagnosis).

She was referred back to the gastroenterology service for evaluation of hepatomegaly. Her infectious disease workup was negative for HIV, Hepatitis B and C, and Syphilis. Rectal biopsy

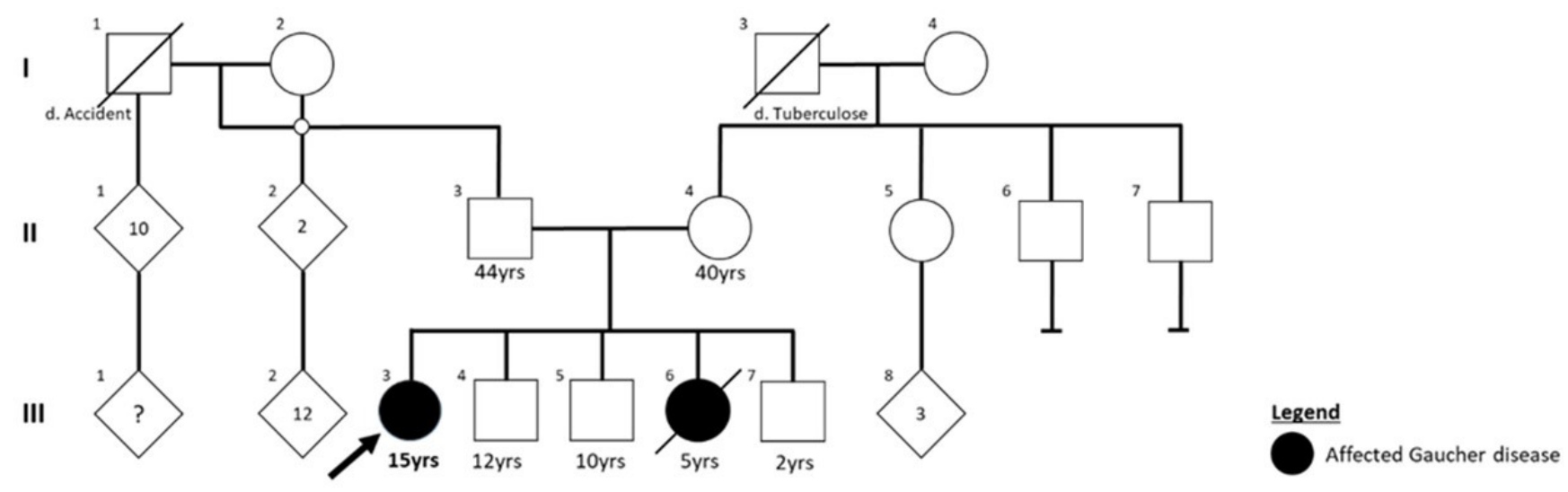

Figure 1. Family history. Members III3 and III6 were identified as having GD. III6 was diagnosed by histological examination in 2016. 
was negative for Schistosoma eggs. No portal hypertension was noticed on abdominal ultrasound.

Due to persistent clinical symptoms and progressive hepatomegaly, a liver biopsy was performed one year later when she was 14 years old. The pathology report suggested a lysosomal disease, most probably GD.

The patient was referred to the medical genetics service at $\mathrm{HCM}$ when she was 15 years of age and a family tree was constructed (Figure 1). Additional tests were ordered, including blood biochemistry, protein electrophoresis, and magnetic resonance imaging to assess brain, skeleton and abdominal, bone density and mutational analyses. At that time her weight was $34 \mathrm{~kg}$, height was $140 \mathrm{~cm}$, with a body mass index of 17.3 $\mathrm{kg} / \mathrm{m}^{2}$. Her school performance was above average.

\section{Pathology}

\section{Clinical Pathology}

Laboratory exams had shown a typical evolution of GD with progressive anemia, thrombocytopenia, and increased ferritin levels (Table 1). Platelet counts have increased after splenectomy was performed in January 2018.

\section{Anatomic Pathology}

Histologic examination of the liver and spleen biopsies demonstrated infiltration of splenic parenchyma and portal area of the liver by enlarged histiocytic cells with granular cytoplasm with characteristic "wrinkled tissue paper" or "crumpled silk" appearance, and eccentric nuclei (Figure 2-A). Histiocytic cells were strongly positive for periodic acid Schiff (PAS) staining and for CD68 in immunohistochemistry (Figure 2-B).

\section{Imaging (Resonance Magnetic Nuclear)}

\section{Liver}

Marked hepatomegaly with the presence of T2-weighted images of central predominance and perihilar disposition, representative of subacute inflammatory process and fibrosis. No areas of hepatic ischaemia (Figure 3).

\section{Musculoskeletal System}

The signal changes along the bone, with emphasis on the femoral head and peri-trochanteric region, with extension to the femoral epiphyses and minor trochanters (Figure 4). No areas of osteonecrosis. Lumbar bone marrow load score: 5 .

Progressive T3 pathological fracture, showing clear collapse of the vertebrae, leading to deformity. A homogeneous and subtle pattern of signal reduction is observed along the vertebral and sacrum bodies without involving the fatty planes adjacent to the basal vascular complex. In addition, degenerative changes Modic type-1, predominant in the lumbar segment of the spine (Figure 5). No evidence of areas of infarction. Bone marrow load score of the spine: $7 / 16$.

\section{Brain}

Discreet brain atrophy is observed in the frontal topography without signs of deposits (Figure 6).

Table 1. Hematological and Biochemistry parameters.

\begin{tabular}{|c|c|c|c|c|c|c|c|c|}
\hline & May 2015 & May 2016 & Dec 2016 & Oct 2017 & Feb 2018 & Sep 2018 & Feb 2020 & Jul 2020 \\
\hline & \multicolumn{4}{|c|}{ Pre-splenectomy period } & \multicolumn{4}{|c|}{ Post-splenectomy period } \\
\hline Hemoglobin (g/dL) & 9.1 & 7 & 7.8 & 7.4 & 10.5 & 12.7 & 11.1 & 11.0 \\
\hline Platelets $(/ \mu \mathrm{L})$ & 127 & 69 & 95 & 48 & 560 & 369 & 500 & 244 \\
\hline Ferritin $(\mathrm{ng} / \mathrm{mL})$ & - & 131 & 348 & 485 & - & - & - & 256.5 \\
\hline Iron $(\mu \mathrm{mol} / \mathrm{L})$ & - & - & - & - & - & - & 3.57 & 3.57 \\
\hline $\mathrm{TIBC}(\mu \mathrm{mol} / \mathrm{L})$ & - & - & - & - & - & - & 56.7 & 67 \\
\hline AST (IU/L) & - & 51 & - & 72 & 56 & 73 & 51.5 & 39 \\
\hline FSH (IU/L) & - & - & - & - & - & - & - & 4.56 \\
\hline LH (IU/L) & - & - & - & - & - & - & - & 7.43 \\
\hline E2 (IU/L) & - & - & - & - & - & - & - & 64.15 \\
\hline Free T3 (ng/mL) & - & - & - & - & - & - & - & 1.39 \\
\hline Free T4 $(\mu \mathrm{g} / \mathrm{dL})$ & - & - & - & - & - & - & - & 9.07 \\
\hline $\mathrm{TSH}(\mu \mathrm{IU} / \mathrm{mL})$ & - & - & - & - & - & - & - & 2.87 \\
\hline Protein electrophoresis & - & - & - & - & \multicolumn{4}{|c|}{ Polyclonal hypergammaglobulinemia } \\
\hline
\end{tabular}

Legend: AST-Aspartate aminotransferase, E2-Estradiol, FSH-Follicle stimulating hormone, LH-Luteinizing hormone, T3-Triiodothyronine, T4- Thyroxine, TIBC-Total iron binding capacity, TSH-Thyroid stimulating hormone 


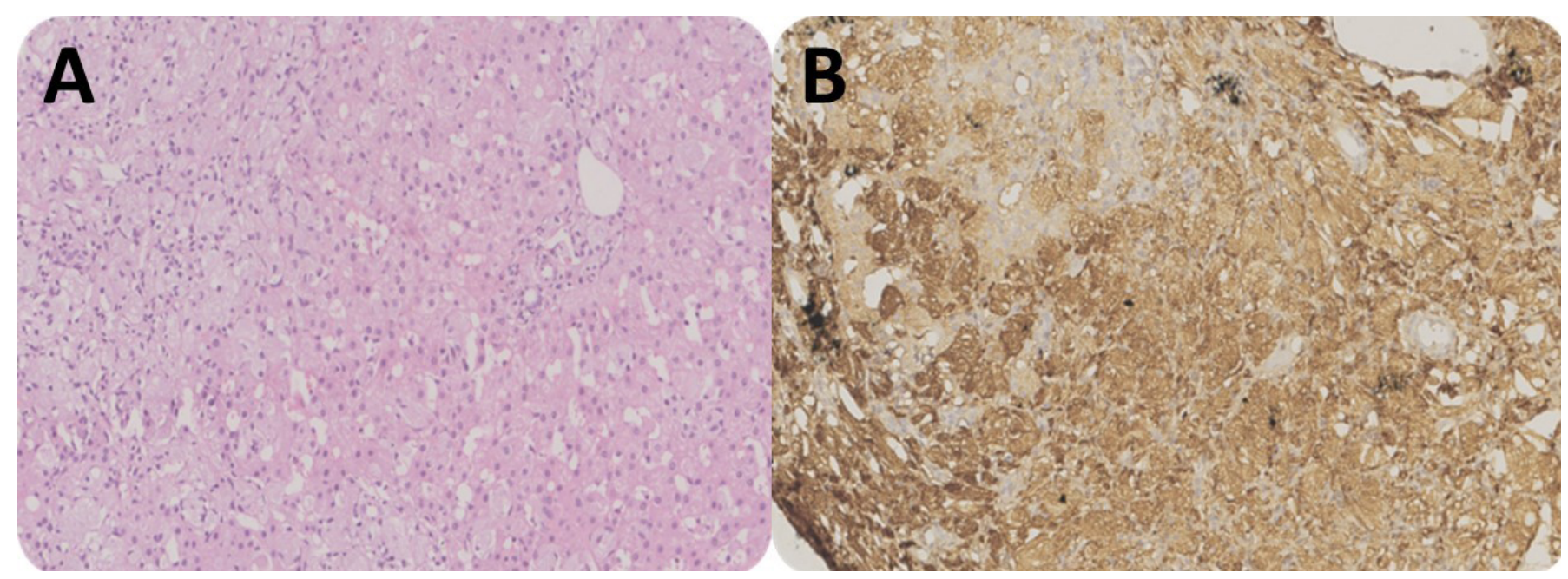

Figure 2. Histopathological appearance of liver involvement in GD. A: Gaucher cells infiltrating the liver parenchyma (200X, H\&E stain). B: "Gaucher cells" strongly positive for CD68 on immunostaining (200X).

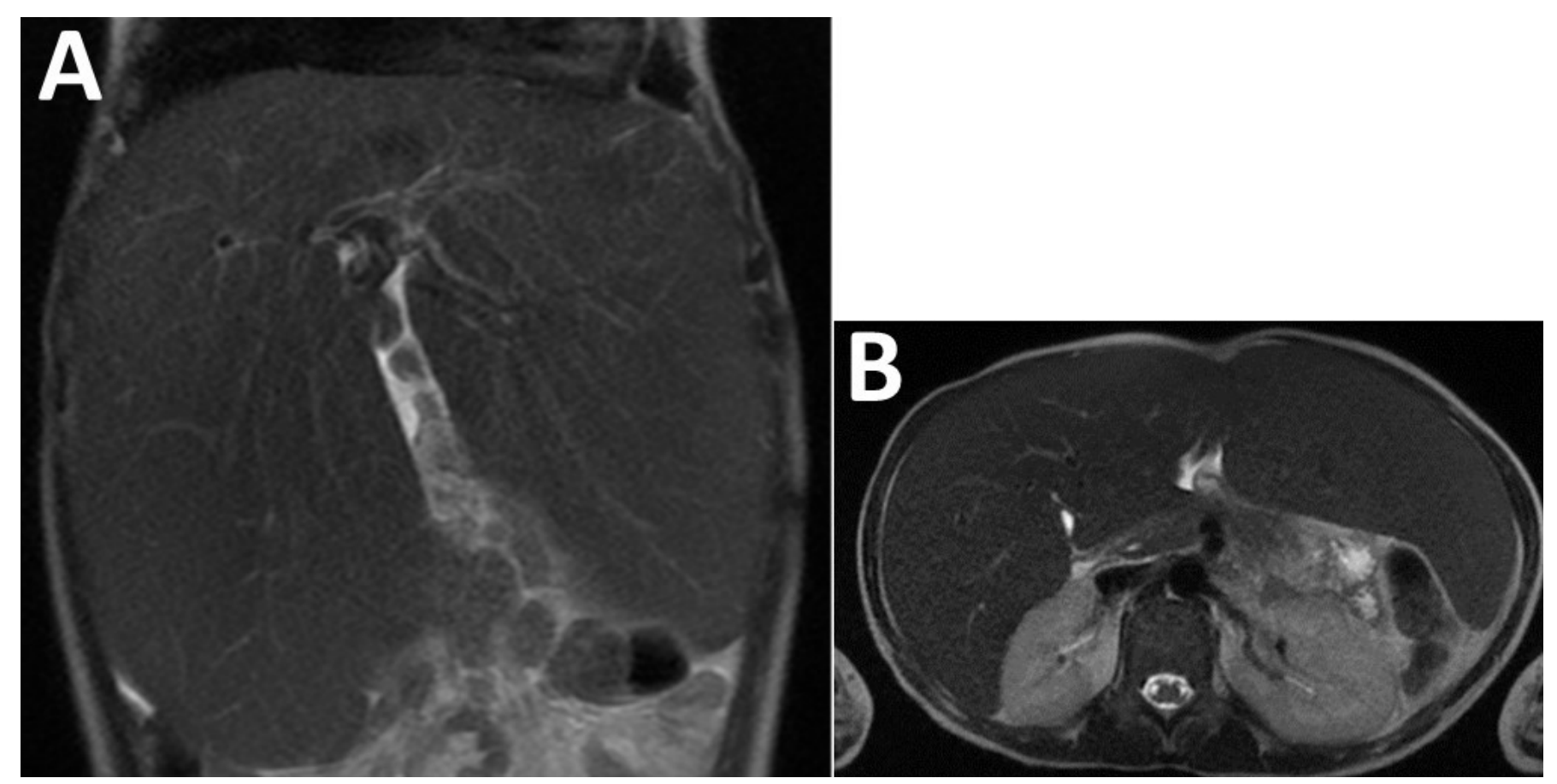

Figure 3. Liver with signs of fibrosis. A: Coronal T2-weighted SSFSE image. B: Axial T2-weighted SSFSE image.

\section{Biochemical Investigation}

Dried blood spots were collected for biochemical analysis $\beta$-glucocerebrosidase activity and quantitation of lyso-Gb1 biomarker, using liquid chromatography/tandem mass spectrometry (LC-MS/MS) (Xevo TQ-S micro, Waters ${ }^{\circledast}$ Corporation) at the Biodiscovery Research Group from Hospital das Clínicas de Porto Alegre, Brazil. Results were notable for a low activity of $\beta$-glucocerebrosidase $0.223 \mathrm{nmol} / \mathrm{h} / \mathrm{ml}$ (cut-off for Gaucher patients $<0.98 \mathrm{nmol} / \mathrm{h} / \mathrm{ml}$ ) and high levels of lysoGb1 $0.43 \mu \mathrm{g} / \mathrm{mL}$ (Control $<0.003 \mu \mathrm{g} / \mathrm{ml}$ and Gaucher patients $>0.06 \mu \mathrm{g} / \mathrm{ml})$.

\section{Molecular Genetics Investigation}

Genomic DNA analysis was isolated from dried blood spots and performed by sequencing on the Ion S5 System platform (Thermo Scientific ${ }^{\mathrm{TM}}$ ) using a customized panel (Ion AmpliSeq ${ }^{\mathrm{TM}}$ Thermo Scientific ${ }^{\mathrm{TM}}$ ), including the GBA1 gene. Data were analysed on the bioinformatics platform Ion Torrent Suite and Ion Reporter (Thermo Scientific ${ }^{\mathrm{TM}}$ ) v 5.10.5.0. The genetic analysis of the patient's DNA showed the pathogenic variant c.259C>T p.Arg87Trp (R48W) in homozygosis in the GBA1 gene (Table 2). 
Table 2. Variants found along the sequence.

\begin{tabular}{lccccccc}
\hline Locus & Reference & Genotype & Coverage & Location & cDNA & Protein & dbSNP \\
\hline Chr1:155209725 & $\mathrm{G}$ & $\mathrm{A} / \mathrm{A}$ & 162 & Exon 4 & c.259C>T & p.Arg87Trp & Rs1141814 \\
\hline
\end{tabular}

Legend: Arg-Arginine, Chr-Chromosome, cDNA-complementary DNA, dbSNP- Single Nucleotide Polymorphism Database, TrpTryptophan

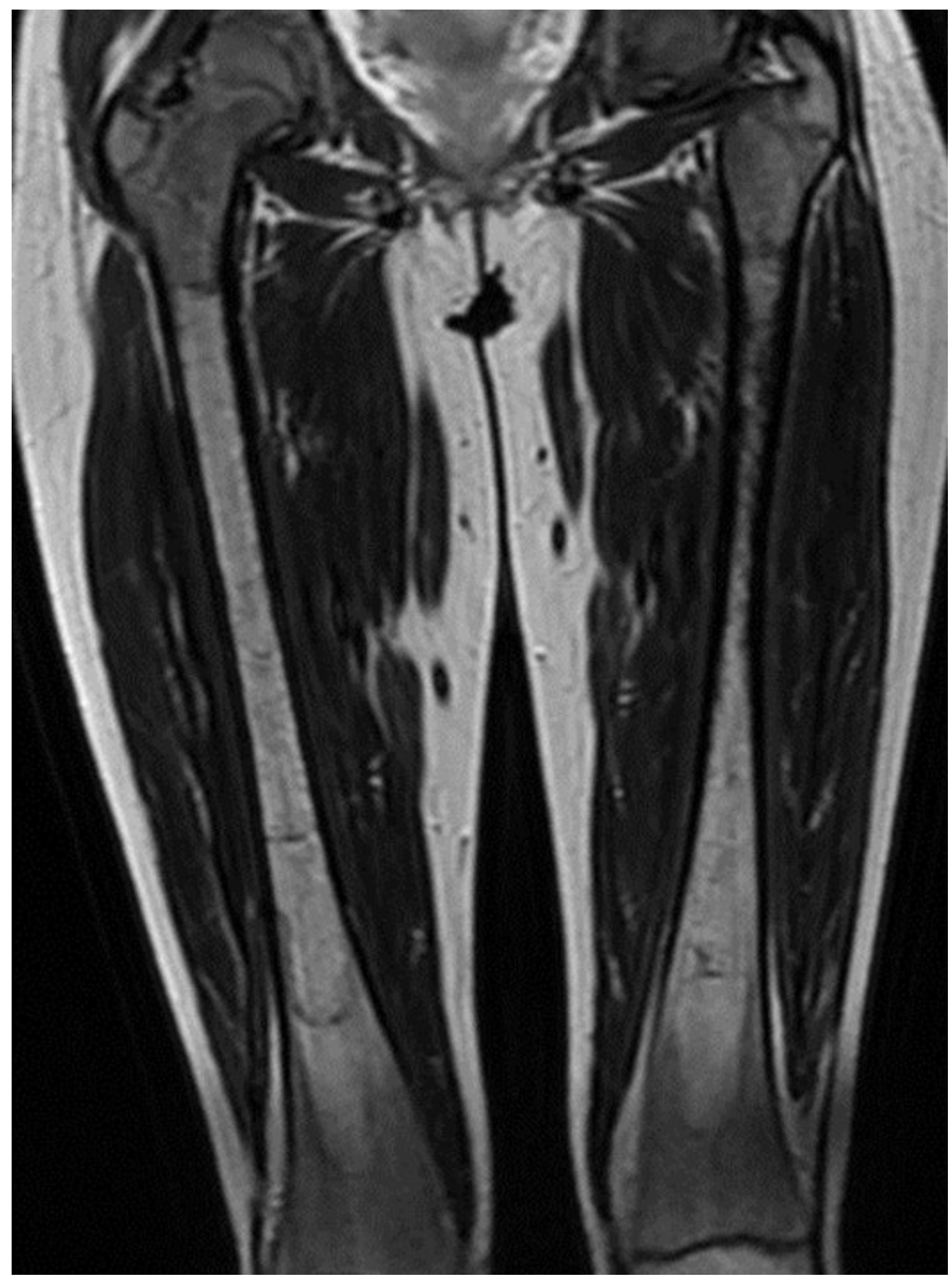

Figure 4. Femur with changes along the bone, Coronal T2-weighted FSE Image. 


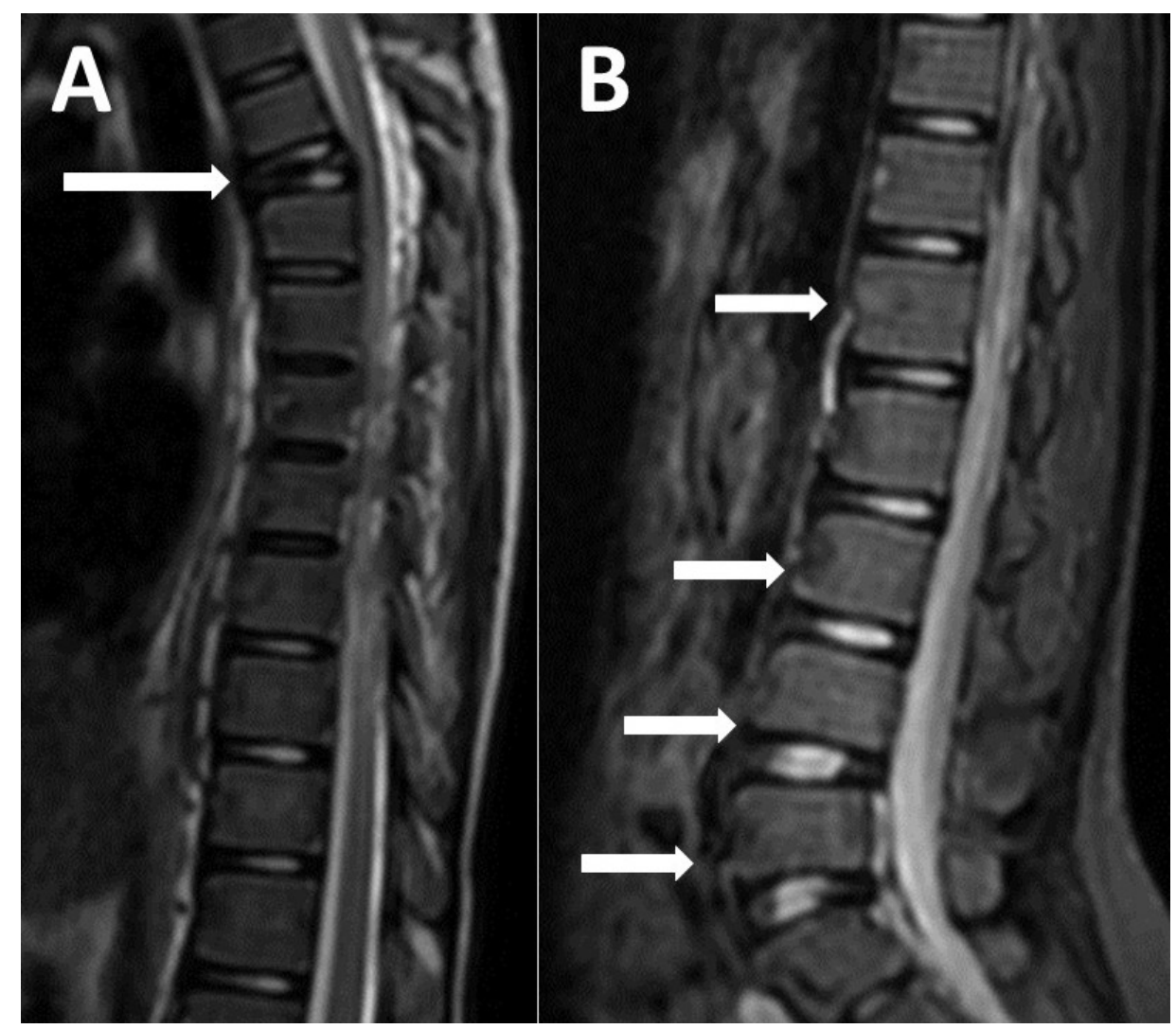

Figure 5. Spine. A - Pathological fracture at T3 vertebra, sagittal STIR image of the cervical spine. B - Subtle signal reduction showed in the vertebrae and Modic type 1 changes, sagittal STIR image of the lumbar spine.
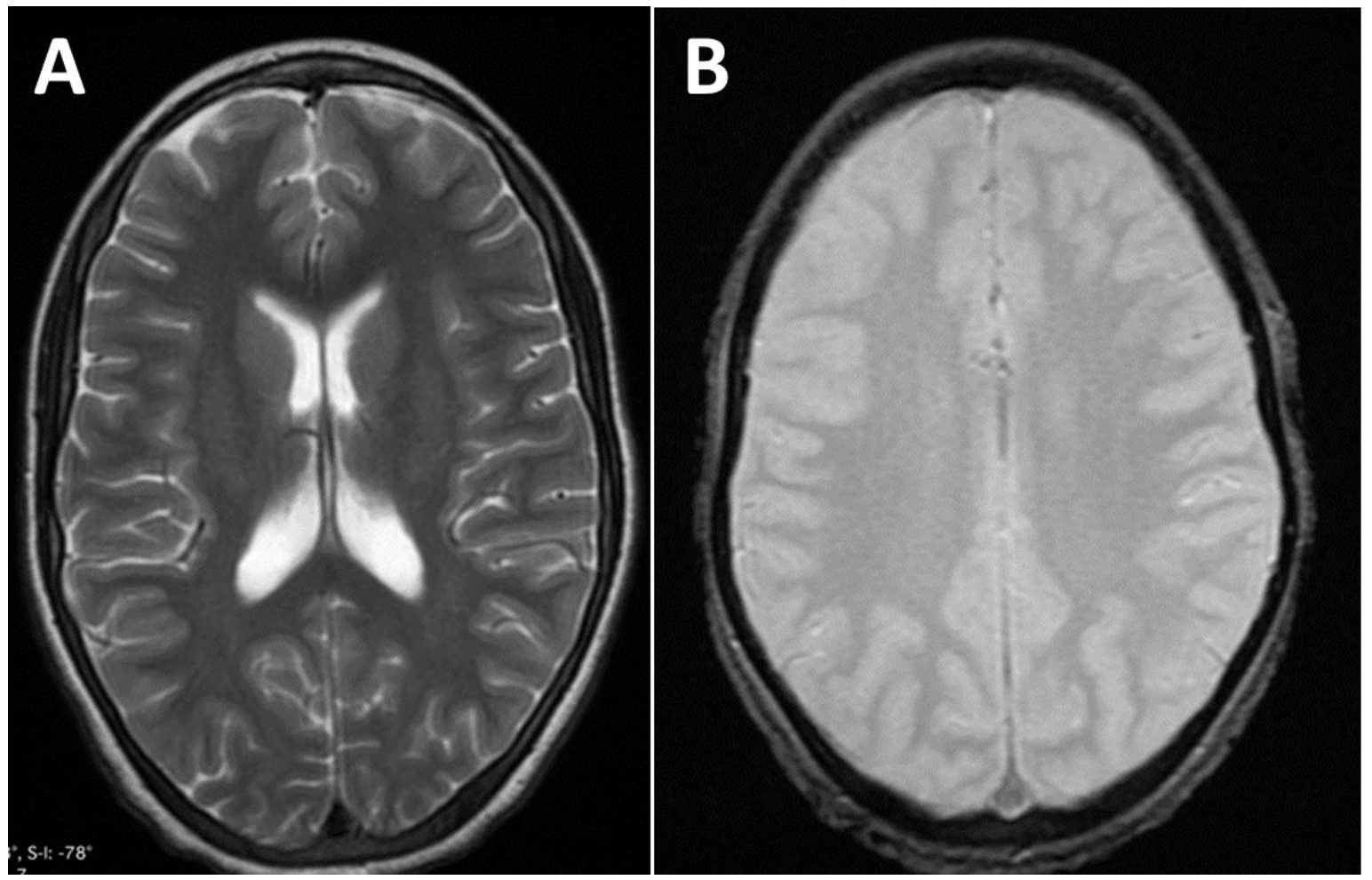

Figure 6. Brain mass without signs of deposit. A: Axial T2-weighted frFSE image. B: Axial T2-weighted GRE image. 


\section{Discussion and Conclusion}

The patient's diagnosis led to suspicion that her sister who had a similar initial presentation and died at the age of 5 after splenectomy surgery also had GD. The sister never had any genetic testing performed. Additional retrospective analyses at the anatomic pathology database at HCM identified another 3 cases of probable GD diagnosed through biopsy and classic histological analysis (haematoxylin-eosin and Periodic Acid Shift stain). All were females with ages ranging from 1-12 years at presentation (one patient without age in the database).

The suspicion of a genetic condition only arose with the second similar case in the family. The diagnosis was suspected after reviewing liver and spleen biopsy results that had cells with cytoplasmic accumulation suggestive of GD. Confirmatory biochemical and genetic testing was performed at a specialised laboratory outside of Mozambique, using dried blood spots.

This patient had clinical and laboratory findings that are common in GD, including bone marrow and iron metabolism dysfunction as anaemia, thrombocytopenia, bleeding episodes[2,6], high levels of ferritin[19], hypersplenism and abdominal distension $[2,20]$, and hypergammaglobulinemia[5,6]. Moreover, growth failure affecting height and weight was observed, but results of endocrine tests assessing thyroid and gonadotrophic axis showed no abnormalities suggestive of a hormonal disorder[21].

The patient displayed normal development and advanced academic performance for age, without evidence of peripheral or central nervous system impairments. These findings are suggestive of GD type 1, which does uncommonly present with neurologic or developmental abnormalities[12,22].

She also had radiographic studies consistent with the diagnosis of GD. As reported in other cases, there were changes in the femoral head and femoral diaphysis that could evolve to osteonecrosis and put her at risk for pathologic fractures[23,24]. She also had classic lesions on MRI of the axial skeleton: pathologic fracture of the T3 vertebra, subtle intensity increased and compressed vertebrae, and a pseudofusion of discs between T3. All these findings constitute severe bone disease with infiltration of the bone marrow. These are classic imaging findings and are part of the natural history of GD[25]. On MRI there was also hepatic enlargement with tightening of the porta region[2,20]. And, the brain mass does not show deposit disease.

The biochemical results observed (substrate accumulation and enzyme deficiency, namely, lyso-Gb1 $0.43 \mu \mathrm{g} / \mathrm{ml}$ and $\beta$-glucocerebrosidase $0.223 \mathrm{nmol} / \mathrm{h} / \mathrm{ml}$ ) correlated with physiopathology, clinical features and other laboratory results, supporting the diagnosis of $\mathrm{GD}[4,8,16]$. On $G B A 1$ genotyping, the patient was found to be homozygous for the variant R48W (p.Arg87Trp). This variant was previously described in the literature and, according to ACMG guidelines[32], it is pathogenic leading to reduced enzyme activity[26-28].
There were delays in her referral and evaluation that reflect weaknesses in symptom recognition and intra-hospital communication and coordination of care. There were also early failures in symptom recognition and linking her presentation to the family history, which was notable for a sister who died with similar symptoms. Ultimately, she was diagnosed via a multidisciplinary team of specialists, and this approach is recommended for future suspects of genetic disorders.

Mozambique is a low-income country, with most of the population depending on healthcare provided by the National Health System, which is largely underfunded. The budget allocated to the Ministry of Health, mainly mitigates public health burdens as malnutrition, tuberculosis, HIV and malaria[17,18]. The Mozambican NHS has a patient referral system that comprises four levels, from primary care to the most differentiated level. And the Ministry of Health recognizes gaps in its practical operation[29]. These gaps represent an additional challenge for health professionals when faced with cases of patients and families with rare diseases. The introduction of a diagnosis and treatment protocol for GD in the national health system is not feasible at this moment due to the high cost to keep these patients under follow-up and treatment due to underfunding of the health system[30]. Thus, even quaternary centres such as HCM do not have the infrastructure or resources to diagnose and treat rare genetic disorders such as GD.

In summary, this patient was diagnosed with GD type 1 being homozygous for p.Arg87Trp (R48W) GBA1 mutation, after presenting with significant bone and visceral disease, and with hematological features[26,27,31]. The patients presents with a moderate to marked degree of disease severity, although the evaluation is based on RMN and clinical findings. The present case represents the first in Mozambique to be studied completely until diagnosis by genotyping. In Africa, 2 alleles of this variant have been published, with no record of occurrence in homozygosis and the estimated allelic frequency is 0.000123 .

To manage the case there is a need to introduce specific treatment to control the progression of the disease. The International Gaucher Alliance (IGA) was contacted and an application was submitted to enable the patient to receive specific treatment from a caritative program on a compassionate use basis. She is currently being followed at six-month intervals in the Genetics service at HCM to monitor for disease complications[5].

\section{Acknowledgements}

The study group address thanks to the followings: Gastroenterology Service, Paediatric Surgery Service, Paediatric Haematology-Oncology Service, and Radiology and Imaging Service for clinical to Óscar Moreno and Edite Mambo performing imaging exams. LSD Brazil Network, Medical Genetics Service, Hospital das Clínicas de Porto Alegre for the valuable contribution on biochemistry identification and genotyping. International Gaucher Alliance Miss Tanya Collin- 
Histed for doing advocacy among the pharmaceutical industries to provide treatment. Dr Guilherme Perini to transport the sample from Mozambique to Brazil. Dr W. Chris Buck assisted with English language revision of the manuscript. Furthermore, we thank the patient and parents.

\section{Ethics Approval and Consent to Participate}

Ethical approval was obtained from the Institutional Research Bioethics Committee of the Faculty of Medicine of the University of Eduardo Mondlane and Hospital Central de Maputo, reference number CIBS FM\&HCM/38/2020. Written consent was obtained from the parents and assent given by the patient to participate and publication.

\section{Authors' Contributions}

FP: report case design, data collection, data analysis, manuscript drafting.

FF, LM, EN, MI, AJ, ACBF, FK: data collection, data analysis. $\mathrm{RG}, \mathrm{FF}$ : manuscript revision.

\section{Declaration of Conflicting Interests}

The authors declared no potential conflicts of interest with respect to the research, authorship, and/or publication of this article.

\section{References}

1. Poswar FO, Vairo F, Burin M, et al. Lysosomal diseases: Overview on current diagnosis and treatment. Genet $\mathrm{Mol}$ Biol. 2019;42(1 suppl 1):165-177. doi: 10.1590/1678-4685GMB-2018-0159

2. Jaffe DH, Flaks-Manov N, Benis A, et al. Populationbased cohort of 500 patients with Gaucher disease in Israel. BMJ Open. 2019;9(1):e024251. doi: 10.1136/ bmjopen-2018-024251

3. Mistry PK, Lopez G, Schiffmann R, Barton NW, Weinreb NJ, Sidransky E. Gaucher disease: Progress and ongoing challenges. Mol Genet Metab. 2017;120(1-2):8-21. doi: 10.1016/j.ymgme.2016.11.006

4. Fateen E, Abdallah ZY. Twenty-five years of biochemical diagnosis of Gaucher disease: the Egyptian experience. Heliyon. 2019;5(10):e02574. doi: 10.1016/j.heliyon.2019. $\mathrm{e} 02574$

5. Baris HN, Cohen IJ, Mistry PK. Gaucher disease: The metabolic defect, pathophysiology, phenotypes and natural history. Pediatr Endocrinol Rev. 2014;12:72-81. https:// www.ncbi.nlm.nih.gov/pmc/articles/PMC4520262/
6. Stirnemann J, Belmatoug N, Camou F, et al. A Review of Gaucher Disease Pathophysiology, Clinical Presentation and Treatments. Int J Mol Sci. 2017;18(2):441. doi: 10.3390/ ijms18020441.

7. Brady R, Kanfer J, Shapiro D. Evidence of an enzymatic deficiency in Gaucher's disease. Biochem Biophys Res Commun. 1965; 8:221-225. doi: 10.1016/0006$291 \times(65) 90743-6$

8. Cullufi P, Tabaku M, Beetz C, Tomori S, Velmishi V, Gjikopulli A, Bauer P, Wirth S, Rolfs A. Comprehensive clinical, biochemical and genetic screening reveals four distinct GBA genotypes as underlying variable manifestation of Gaucher disease in a single family. $\mathrm{Mol}$ Genet Metab Reports. 2019;21:100532. doi: 10.1016/j. ymgmr.2019.100532

9. Mehta A. Epidemiology and natural history of Gaucher's disease. Eur J Intern Med. 2006;17(suppl):S2-S5. doi: 10.1016/j.ejim.2006.07.005

10. Biegstraaten M, Cox TM, Belmatoug N, et al. Management goals for type 1 Gaucher disease: An expert consensus document from the European working group on Gaucher disease. Blood Cells Mol Dis. 2018;68:203-208. doi: 10.1016/j. bcmd.2016.10.008

11. Regenboog M, van Dussen L, Verheij J, et al. Hepatocellular carcinoma in Gaucher disease: an international case series. J Inherit Metab Dis. 2018;41(5):819-827. doi: 10.1007/ s10545-018-0142-y

12. Mullin S, Hughes D, Mehta A, Schapira AHV. Neurological effects of glucocerebrosidase gene mutations. Eur J Neurol. 2019;26:388-e29. doi: 10.1111/ene.13837

13. Aflaki E, Westbroek W, Sidransky E. The Complicated Relationship between Gaucher Disease and Parkinsonism: Insights from a Rare Disease. Neuron. 2017;93:737-746. doi: 10.1016/j.neuron.2017.01.018

14. Avenali M, Blandin F, Cerri S. Glucocerebrosidase Defects as a Major Risk Factor for Parkinson's Disease. Front Aging Neurosci. 2020;12:97. doi: 10.3389/fnagi.2020.00097

15. Noya M, Andrade-Campos M, Irun P, López de Frutos L, López-Fernandez M, Giraldo P. Gaucher disease and chronic myeloid leukemia: first reported patient receiving enzyme replacement and tyrosine kinase inhibitor therapies simultaneously. Clin Case Rep. 2018;6(5):887-892. doi: $10.1002 /$ ccr3.1460

16. Bobillo Lobato J, Jiménez Hidalgo M, Jiménez Jiménez L. Biomarkers in Lysosomal Storage Diseases. Diseases. 2016;4(4):40. doi: 10.3390/diseases 4040040 
17. United Nations Development Programme. Human development report 2019: beyond income, beyond averages, beyond today. http://hdr.undp.org/sites/default/files/ hdr2019.pdf

18. UNICEF. Mozambique Health Budget Brief 2018. https://www.unicef.org/mozambique/sites/unicef.org. mozambique/files/2019-04/2018-budget-brief-health.pdf.

19. Lefebvre T, Reihani N, Daher R, et al. Involvement of hepcidin in iron metabolism dysregulation in Gaucher disease. Haematologica. 2018;103(4):587-596. doi: 10.3324/ haematol.2017.177816

20. Sagar A, Pathak A, Bohra V, Nair V. An unusual case of massive hepatosplenomegaly. Indian J Endocrino Metab. 2013;17(1):179-181. doi: 10.4103/2230-8210.107881

21. Gary SE, Ryan E, Steward AM, Sidransky E. Recent advances in the diagnosis and management of Gaucher disease. Expert Rev Endocrinol Metab. 2018; 13(2):107-118. doi: 10.1080/17446651.2018.1445524

22. Biegstraaten M, Wesnes KA, Luzy C, et al. The cognitive profile of type 1 Gaucher disease patients. J Inherit Metab Dis. 2012;35(6):1093-1099. doi: 10.1007/s10545-0129460-7

23. Simpson WL. Imaging of gaucher disease. World J Radiol. 2014;6(9):657-68. doi: 10.4329/wjr.v6.i9.657

24. Degnan AJ, Ho-Fung VM, Ahrens-Nicklas RC, Barrera CA, Serai SD, Wang DJ, Ficicioglu C. Imaging of nonneuronopathic Gaucher disease: recent advances in quantitative imaging and comprehensive assessment of disease involvement. Insights Imaging. 2019;10(1):70. doi: 10.1186/s13244-019-0743-5

25. Terk MR, Esplin J, Lee K, Magre G, Colletti PM. MR imaging of patients with type 1 Gaucher's disease: relationship between bone and visceral changes. AJR Am J Roentgenol. 1995;165(3):599-604. doi: 10.2214/ajr.165.3.7645477

26. National Center for Biotechnology Information. ClinVar; [VCV000004321.2], https://www.ncbi.nlm.nih.gov/clinvar/ variation/VCV000004321.2

27. UniProt. P04062 (GLCM_HUMAN). https://www.uniprot. org/uniprot/P04062\#section_seq

28. OMIM. ${ }^{\star} 606463$ GLUCOSIDASE, BETA, ACID; GBA. https://www.omim.org/entry/606463

29. REPÚBLICA DE MOÇAMBIQUE - MINISTÉRIO DA SAÚDE. Plano Estratégico do Sector da Saúde, PESS 20142019. 2013. https://www.misau.gov.mz/index.php/planosestrategicos?download=132:plano-estrategico-do-sectorda-sade-2014-2019

30. Beutler E. The treatment of Gaucher disease in countries with limited health care resources. Indian J Hum Genet. 2005;11(3):121-127. https://tspace.library.utoronto.ca/ bitstream/1807/5945/1/hg05024.pdf

31. OMIM. \# 230800 GAUCHER DISEASE, TYPE I. https:// www.omim.org/entry/230800

32. VarSome. chr1-155209725-G-A (GBA:p.R87W). https:// varsome.com/variant/hg19/chr1-155209725-G-A. 